\title{
Downregulated CDK10 expression in gastric cancer: Association with tumor progression and poor prognosis
}

\author{
YANJIE YOU*, FEIHU BAI*, ZHENGCAI YE, NAN ZHANG, LI YAO, YUANYUAN TANG and XIMEI LI \\ Department of Gastroenterology, Ningxia Hui Autonomous Region People's Hospital, \\ Yinchuan, Ningxia Hui Autonomous Region 750021, P.R. China
}

Received August 18, 2017; Accepted January 12, 2018

DOI: $10.3892 / \mathrm{mmr} .2018 .8662$

\begin{abstract}
The tumor suppressor characteristics of cyclin-dependent kinase 10 (CDK10) in nasopharyngeal carcinoma and breast cancer have been previously demonstrated. In the present study the expression status of CDK10 and its prognostic significance in gastric cancer was determined, as well as its role in cell proliferation and invasion. Immunoblot analysis revealed that CDK10 protein expression was notably decreased in gastric cancer compared with normal tissues. Immunohistochemistry demonstrated that the loss of CDK10 expression, which was observed in $50.8 \%$ of primary gastric cancer tissues $(n=128)$, significantly correlated with advanced tumor stage $(\mathrm{P}<0.001)$, frequent lymph node metastasis $(\mathrm{P}<0.001)$, distant metastasis $(\mathrm{P}=0.013)$, tumor differentiation $(\mathrm{P}=0.004)$ and unfavorable overall survival $(\mathrm{P}<0.001)$. Multivariate analysis suggested that CDK10 expression may serve as an independent prognostic predictor $(\mathrm{P}=0.001)$ for the progression of gastric cancer. In addition, ectopic CDK10 expression inhibited gastric cancer cell proliferation, migration and invasion, while knockdown of CDK10 promoted these phenotypes. Collectively, the results of the present study indicated that CDK10 expression may serve as a novel prognostic biomarker that holds therapeutic promise for gastric cancer.
\end{abstract}

\section{Introduction}

Gastric cancer is curable if it is diagnosed at early stages, however, most patients are diagnosed at a late stage when

Correspondence to: Professor Feihu Bai or Dr Yanjie You, Department of Gastroenterology, Ningxia Hui Autonomous Region People's Hospital, 301 Zhengyuan North Road, Yinchuan, Ningxia Hui Autonomous Region 750021, P.R. China

E-mail: baifeihu@sohu.com

E-mail: youyanjie@163.com

*Contributed equally

Key words: cyclin-dependent kinase 10, gastric cancer, immunohistochemistry, prognosis the current cancer regimen is limited (1-3). Although surgery combined with chemotherapies has been used to treat advanced gastric cancer, the overall 5-year survival rate is less than $24 \%(3,4)$. Over the past decades, numerous studies have been focusing on the molecular mechanisms of gastric cancer. Alterations of certain gene expression and genetic variation have been discovered in gastric cancer (5). However, the exact molecular mechanisms underlying gastric cancer initiation and progression is still not well understood, which prevents the development of personalized and suitable cancer therapy strategies. Therefore, it is critical to discover reliable molecular biomarkers for gastric cancer, which can be used for prognosis and therapeutic targets.

We found that cyclin-dependent kinase 10 (CDK10, also called PISSLRE) is one of the targets with potential prognostic impact and great therapeutic promise. In human nasopharyngeal carcinoma, CDK10 gene promoter is often hypermethylated resulting in its silence, while restoration of CDK10 expression may strongly suppress the malignant behaviors of cancer cells (6). Our previous report revealed that CDK10 expression levels are significantly decreased in breast cancer and correlated with patient survival rates $(7,8)$.

CDKs currently hold great promise for anti-tumor therapeutics and are been considered as potential molecular targets for cancer therapy. CDK10, a cdc2-related kinase, is essential for cell cycle progression from the G2 to M phase (9-11). In breast cancer, the resistance to endocrine therapies is determined by CDK10, the silencing of which leads to cancer cell reliance lost upon estrogen signaling $(12,13)$.

The expression of CDK10 is observed in various normal tissues, whereas it is often decreased or absent in cancers $(7,12,14,15)$. Furthermore, overexpression of CDK10 increases the sensitivity of hepatocellular carcinoma and biliary tract cancer cells to chemotherapy $(14,15)$. Together with our reports, these encouraging findings suggest that CDK10 may be a tumor suppressor candidate, which contributes to prognosis as well as offers new personalized therapeutic strategies for cancers. To the best of our knowledge, the expression pattern, clinical relevance and biological functions of CDK10 in gastric cancer are not well understood. To this aim, we firstly evaluated CDK10 expression status and its clinical significance in gastric cancer. We then determined the tumor suppressing functions of CDK10 with respect to cancer cell proliferation, migration and invasion. 


\section{Materials and methods}

Patients and tissue samples. We randomly collected patient samples from the Cancer Hospital of Shantou University Medical College. 128 formalin-fixed paraffin-embedded (FFPE) specimens were obtained from patients with gastric cancer undergoing curative surgery between 2000 and 2005 (median age, 61 years; range, 35-84 years). The median follow-up period was 37 months (range, 1-80 months) from the date of surgery. Additionally, cancer tissues $(n=20)$ and their matching noncancerous tissues $(n=20)$, for immunoblot assays, were immediately snap frozen in liquid nitrogen and kept at $-80^{\circ} \mathrm{C}$ until further use. Of note, they were harvested from another independent cohort of patients with gastric cancer who underwent surgery at the same institution between May 2010 and July 2012.

Tumor grade and stage were classified according to the International Union against Cancer (UICC)/American Joint Committee on Cancer (AJCC) pathologic tumor-node-metastasis (TNM) classification, 7th edition (2010). Table I demonstrates the clinicopathologic characteristics for these patients. No patient was found to have undergone preoperative radiotherapy or chemotherapy. The current study conformed to the ethical guidelines of the Declaration of Helsinki and was approved by the Institution Review Board (no. 04-470) of the Cancer Hospital of Shantou University Medical College. Written informed consent was obtained from all patients before sample collection. All samples were coded and data was stored anonymously.

Immunoblot analysis. CDK10 protein levels were examined by immunoblot analysis as described previously $(6-8,16,17)$. $60 \mu \mathrm{g}$ proteins in RIPA lysis buffer were separated by SDS-PAGE and transferred to a PVDF membrane. After one hour incubation in blocking buffer (Tris-buffered saline with $0.1 \%$ Tween and $5 \%$ nonfat dry milk), the membrane was then incubated with a rabbit polyclonal antibody against CDK10 (catalog no. ab72710; dilution, 1:500; Abcam, Cambridge, MA, USA), followed by a horseradish peroxidase-conjugated goat anti-rabbit immunoglobulin G (IgG; catalog no. sc-2004; dilution, 1:2,000; Santa Cruz Biotechnology, Inc., Dallas, TX, USA). Signals were visualized using an enhanced chemiluminescence kit (GE Healthcare Life Sciences, Little Chalfont, UK) as described by the manufacturer. Anti-GAPDH monoclonal antibody (catalog no. ab9485; dilution, 1:2,500; Abcam) was used to assure equal loading of protein. Quantification of the intensity of CDK10 in the immunoblot was conducted using the Bio-Rad Quantity One quantitation software (20), with the ratio between the tumors and the paired noncancerous tissues identified as being less than two folds, suggesting decreased CDK10 expression.

Immunohistochemistry and staining evaluation. We performed immunohistochemical staining to detect CDK10 expression using a standard EnVision complex method as described previous $(7,14,17)$. 4- $\mu$ m sections were cut from FFPE specimens and then processed with deparaffinization and rehydration. The endogenous peroxidase activity was blocked with $0.3 \%$ hydrogen peroxide for $30 \mathrm{~min}$ at room temperature. After antigen retrieval, tissue sections were incubated with a rabbit polyclonal anti-CDK10 antibody (catalog no. ab72710; dilution, 1:50; Abcam), after which immunohistochemical staining was conducted by an EnVision antibody complex (anti-Mouse/Rabbit) method using an EnVision ${ }^{\mathrm{TM}}$ Detection kit (ZSGB-BIO, Beijing, China) and 3,3'-diaminobenzidine as the chromogen substrate. As a negative control, the primary antibody was replaced by a normal rabbit IgG.

The staining evaluation was carried out as follows (7): Ten 400x microscopic fields per slide were random selected and evaluated by two independent pathologists. CDK10 immunostaining was determined using a semi-quantitative approach combining the percentage of positive cells and the staining intensity. The mean percentage of stained cells was scored as follows: $0,0 \% ; 1,1-25 \% ; 2,26-50 \% ; 3,51-75 \%$; and 4, 76-100\%. The staining intensity was classified as follows: 0 , Absent; 1, weak staining; 2, moderate staining; and 3, strong staining. We summed up the intensity and extent scores as the final staining score. For the purpose of statistical evaluation, we grouped the tumor samples with a final staining score of $<3$ into negative CDK10 expression and those with scores $\geq 3$ into positive CDK10 expression.

Cell culture and transfection. Two established gastric cancer cell lines (HGC-27 and SGC-7901) were cultured as described previously $(6,16)$. A recombinant CDK10-expressing plasmid (pcDNA3.1-CDK10) and a pre-designed validated siRNA targeting CDK10 (5'-GUCCCAGUAAAGCCAAUGATT-3' and 5'-UCAUUGGCUUUACUGGGACTT3') were prepared as described previously (6). Cell transfection was conducted using Lipofectamine 2000 reagent (GE Healthcare Life Sciences, Little Chalfont, UK) according to the manufacturer's instructions. Cells were harvested $48 \mathrm{~h}$ post-transfection and used for subsequent experiments described below. The expression levels of CDK10 after transfection was confirmed by immunoblot analysis.

Cell proliferation and colony formation assays. The cell proliferative and cologenic capacities were measured using a 3-(4,5-dimethylthiazol-2-yl)-2 5-diphenyl-2H-tetrazolium bromide (MTT) colorimetric assay and a colony formation (soft agar culture) assay, respectively, according to standard methods described before $(6,16,18)$. Each experiment was conducted three times in replicates of six wells.

Wound healing and invasion assays. The cell motility and invasive capacities were measured using a wound healing assay and a Matrigel invasion chamber assay, respectively, according to standard methods described before $(6,16,18)$. Each experiment was conducted in triplicate wells and repeated three times.

Statistical analyses. Statistical analyses were performed using the SPSS statistical software package (version 17.0; SPSS, Inc., Chicago, IL, USA). A Student's t test was used to evaluate the statistical significance of differences in numerical data. The Pearson $\chi^{2}$ test or Fisher's exact test was used to analyze the correlations between CDK10 expression and clinicopathologic parameters, and the Spearman's rank method was used to obtain the correlation coefficient between variables. Overall survival (OS) rates were generated using the Kaplan-Meier 
Table I. Correlation of CDK10 expression with clinicopathological parameters.

CDK10 expression

\begin{tabular}{|c|c|c|c|c|}
\hline Variable & No. of patients & Negative, n (\%) & Positive, n (\%) & P-value \\
\hline \multicolumn{5}{|l|}{ Age } \\
\hline$\leq 60$ years & 63 & $37(58.7)$ & $26(41.3)$ & 0.077 \\
\hline$>60$ years & 65 & $28(43.1)$ & 37 (56.9) & \\
\hline \multicolumn{5}{|l|}{ Gender } \\
\hline Male & 106 & $51(48.1)$ & $55(51.9)$ & 0.185 \\
\hline Female & 22 & $14(63.6)$ & $8(36.4)$ & \\
\hline \multicolumn{5}{|l|}{ Tumor size } \\
\hline$\leq 5 \mathrm{~cm}$ & 64 & $29(45.3)$ & $35(54.7)$ & 0.216 \\
\hline$>5 \mathrm{~cm}$ & 64 & $36(56.3)$ & $28(43.7)$ & \\
\hline \multicolumn{5}{|l|}{ Histological type } \\
\hline Intestinal & 62 & $35(56.5)$ & $27(43.5)$ & 0.578 \\
\hline Diffuse & 50 & $22(44.0)$ & $28(56.0)$ & \\
\hline Mixed & 16 & $8(50.0)$ & $8(50.0)$ & \\
\hline \multicolumn{5}{|l|}{ Differentiation } \\
\hline Well/moderate & 51 & $18(35.3)$ & $33(64.7)$ & 0.004 \\
\hline Poor & 77 & $47(61.0)$ & $30(39.0)$ & \\
\hline \multicolumn{5}{|l|}{ Stage of tumors } \\
\hline $\mathrm{I} / \mathrm{II}$ & 43 & $11(25.6)$ & $32(74.4)$ & $<0.001$ \\
\hline III/IV & 85 & $54(63.5)$ & $31(36.5)$ & \\
\hline \multicolumn{5}{|l|}{ Invasive depth } \\
\hline $\mathrm{T} 1 / \mathrm{T} 2$ & 9 & $3(33.3)$ & $6(66.7)$ & 0.278 \\
\hline T3/T4 & 119 & $62(52.1)$ & 57 (47.9) & \\
\hline \multicolumn{5}{|c|}{ Lymph node metastasis } \\
\hline $\mathrm{N} 0 / \mathrm{N} 1$ & 41 & $11(26.8)$ & $30(73.2)$ & $<0.001$ \\
\hline $\mathrm{N} 2 / \mathrm{N} 3$ & 87 & $54(62.1)$ & 33 (37.9) & \\
\hline \multicolumn{5}{|c|}{ Distant metastasis } \\
\hline M0 & 100 & $45(45.0)$ & $55(55.0)$ & 0.013 \\
\hline M1 & 28 & $20(71.4)$ & $8(28.6)$ & \\
\hline
\end{tabular}

CDK10, cyclin-dependent kinase 10.

method with log rank test. The prognostic significance of clinicopathological variables was determined by univariate and multivariate regression analysis with the Cox hazards model. $\mathrm{P}<0.05$ (two-tailed) was considered to indicate a statistically significant difference.

\section{Results}

Decreased CDK10 protein levels in gastric cancer. Giving that evidenced function of CDK10 as a potential tumor suppressor frequent silenced during tumorigenesis (5-7), we first determined whether CDK10 expression was downregulated in tumors vs. noncancerous tissues. Fig. 1 shows the representative results in a cohort of gastric cancer specimen $(n=20)$ by immunoblot analysis. We found that $85 \%(17 / 20)$ of cancer tissues had lower CDK10 protein levels compared to their matching adjacent noncancerous tissues $(\mathrm{P}<0.001)$.
Correlation of CDK10 expression with clinicopathological parameters. To verify the above observation, we next determined the expression profile and localization of CDK10 protein in 168 gastric cancer specimens by utilizing immunohistochemistry. Positive CDK10 immunostaining was observed primarily in the cytoplasm of neoplastic cells in 50.8\% (65/128) of primary gastric tumors tested (Fig. 2). To better understand the significance of CDK10 expression in gastric cancer, we further assessed the association between CDK10 expression and the clinicopathological parameters. We found that negative CDK10 expression was associated with advanced tumor stage (stage III/IV vs. stage I/II; $\mathrm{P}<0.001$ ), frequent lymph node metastasis (N2/N3 vs. N0/N1; $\mathrm{P}<0.001)$, distant metastasis $(\mathrm{P}=0.013)$ and tumor differentiation (poor vs. well/moderate; $\mathrm{P}=0.004$ ) (Table I). No significant relationship was observed between CDK10 expression and the other clinicopathologic factors such as age, gender, tumor size and invasive depth. Spearman correlation analysis revealed CDK10 expression levels were 


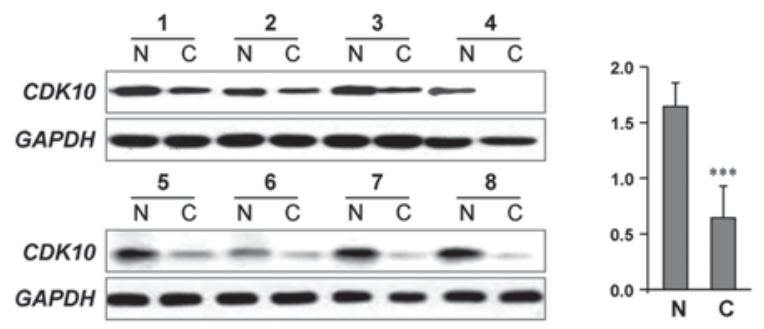

Figure 1. CDK10 protein levels in cancerous and noncancerous tissues. The CDK10 protein levels were assessed by immunoblot analysis in gastric cancer tissues and matching adjacent noncancerous tissues. A quantitative analysis of CDK10 expression normalized by GAPDH is shown in the right panel $(n=20) .{ }^{* * *} \mathrm{P}<0.001$, compared with the $\mathrm{N}$ group. $\mathrm{C}$, cancer tissues; $\mathrm{N}$, noncancerous tissues. CDK10, cyclin-dependent kinase 10.
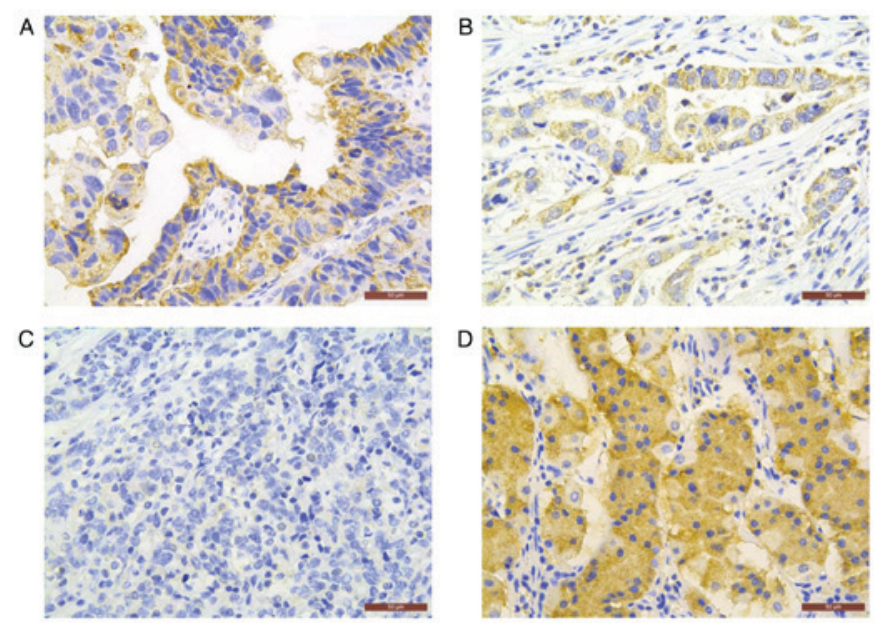

Figure 2. Immunohistochemiscal analysis of CDK10 expression in gastric cancer tissues. (A) Strong CDK10 staining in gastric cancer tissue (B) Moderate CDK10 staining in gastric cancer tissue. (C) Weak CDK10 staining in gastric cancer tissue. (D) Positive CDK10 staining in adjacent noncancerous tissue. Original magnification, $x 400$. Scale bar $=50 \mu \mathrm{m}$. CDK10, cyclin-dependent kinase 10 .

correlated with clinical tumor staging $(r=-0.375 ; \mathrm{P}<0.001)$, lymph node metastasis $(r=-0.349 ; \mathrm{P}<0.001)$, distant metastasis $(r=-0.239 ; \mathrm{P}=0.002)$ and tumor differentiation $(r=-0.288$; $\mathrm{P}<0.001)$. Together, these data demonstrate that a lack of CDK10 expression may correlate with malignant properties mainly relevant to lymph node metastasis and tumor advancement. These observations provide solid evidence that dysregulation of CDK10 expression may contribute to gastric cancer progression.

Association between CDK10 expression and patient survival. Kaplan-Meier survival analyses were performed to investigate the prognostic impacts of CDK10 expression on the outcome of patients with gastric cancer. We found that the OS rate of patients with tumors that expressed CDK10 was higher than that of patients with tumors did not express CDK10 $(\mathrm{P}<0.001)$ (Fig. 3). Further, we examined whether CDK10 expression may be served as a prognostic factor for gastric cancer patients by utilizing univariate and multivariate analyses. On univariate analysis, CDK10 expression $(\mathrm{P}<0.001)$, patient age $(\mathrm{P}=0.039)$, tumor size $(\mathrm{P}<0.001)$, histological type $(\mathrm{P}=0.001)$, stage of tumors $(\mathrm{P}=0.001)$ and lymph node metastasis $(\mathrm{P}<0.001)$ were significantly correlated with an unfavorable OS (Table II).

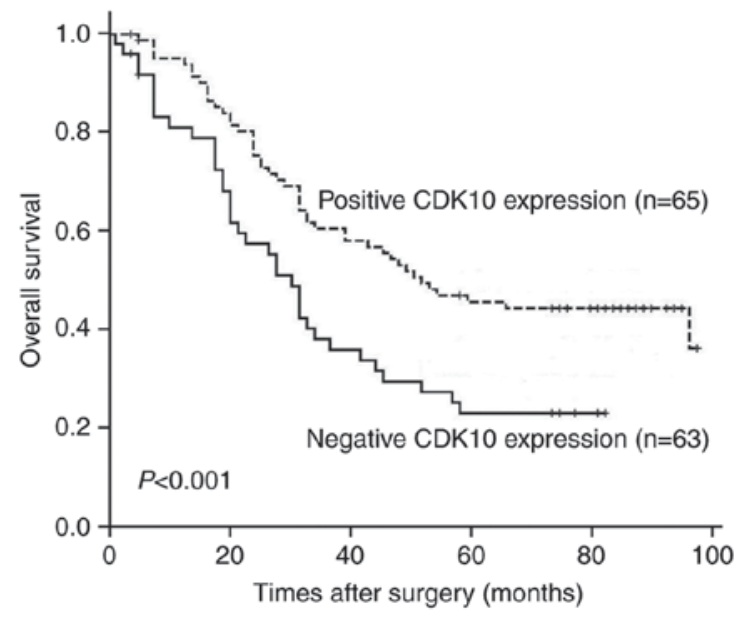

Figure 3. Kaplan-Meier survival curves for overall survival according to CDK10 expression statues. CDK10 expression was significantly associated with prolonged survival for patient with gastric cancer $(\mathrm{P}<0.001)$. CDK10, cyclin-dependent kinase 10 .

After adjusting the prognostic factors that achieved significance in univariate analysis, CDK10 expression $(\mathrm{P}=0.045)$, histological type $(\mathrm{P}=0.004)$ and tumor size $(\mathrm{P}=0.001)$ maintained independent significance for predicting the prognosis of gastric cancer patients.

Ectopic CDK10 expression suppresses gastric cancer cell growth and invasion. To confirm that CDK10 functions as a tumor suppressor in gastric cancer pathogenesis and progression, we examined the effect of CDK10 on cell proliferation by transfecting a CDK10 expression construct into gastric cancer cell lines HGC-27 and SGC-7901. Overexpression of CDK10 was achieved in these two cell lines (Fig. 4A). As evidenced by MTT assays, we found that proliferation rates of these cell lines with CDK10 overexpression were significantly reduced in comparison to the empty vector-transfected control cells (Fig. 4B). Moreover, HGC-27 and SGC-7901 cells exogenously expressing CDK10 produced fewer colonies than their respective control cells (Fig. 4C).

We subsequently investigated whether CDK10 could regulate the metastasis capacities of gastric cancer cells. Confluent HGC-27 and SGC-7901 cells with or without ectopic CDK10 expression were scratched and cell motility was determined. By doing so, we found that ectopic CDK10 expression, in both gastric cell lines, significantly delayed closure of wound area compared to the empty vector controls (Fig. 4D). Next, we examined the invasive ability of these gastric cell lines using a Matrigel assay. As shown in Fig. 4E, CDK10 overexpression let to a significant decrease in invasiveness of both cell lines, as compared with their own controls. All these observations clearly demonstrated that CDK10 expression restoration inhibits the migration and invasion of gastric cancer cells.

Knockdown of CDK10 expression promotes gastric cancer cell growth and invasion. We further assessed the biological effects of silencing CDK10 expression on gastric cancer cells. The efficiency of CDK10 knockdown using a pre-design and validated siRNA was tested by immunoblot analysis (Fig. 5A). We observed that HGC-27 and SGC-7901 cells with CDK10 
Table II. Univariate and multivariate Cox proportional hazards models showing variables that affect overall survival in patients with gastric cancer.

\begin{tabular}{|c|c|c|c|c|}
\hline \multirow[b]{2}{*}{ Variables } & \multicolumn{2}{|c|}{ Univariate analysis } & \multicolumn{2}{|c|}{ Multivariate analysis } \\
\hline & HR (95\% CI) & P-value & HR $(95 \% \mathrm{CI})$ & P-value \\
\hline \multicolumn{5}{|l|}{ Age (years) } \\
\hline$>60$ vs. $\leq 60$ & $0.557(0.319-0.971)$ & 0.039 & $0.686(0.390-1.205)$ & 0.190 \\
\hline \multicolumn{5}{|l|}{ Gender } \\
\hline Male vs. Female & $0.730(0.492-1.258)$ & 0.247 & - & - \\
\hline \multicolumn{5}{|l|}{ Tumor size $(\mathrm{cm})$} \\
\hline$>5$ vs. $\leq 5$ & $3.188(1.964-5.175)$ & $<0.001$ & $2.660(1.588-4.457)$ & 0.001 \\
\hline \multicolumn{5}{|l|}{ Histological type } \\
\hline Poor vs. Well/moderate & $2.676(1.593-4.495)$ & 0.001 & $2.185(1.276-3.740)$ & 0.004 \\
\hline \multicolumn{5}{|l|}{ Differentiation } \\
\hline Intestinal vs. Diffuse & $0.580(0.509-1.117)$ & 0.183 & - & - \\
\hline \multicolumn{5}{|l|}{ Stage of tumors } \\
\hline III/IV vs. I/II & $2.353(1.417-4.107)$ & 0.001 & $1.246(0.677-2.291)$ & 0.480 \\
\hline \multicolumn{5}{|l|}{ Invasive depth } \\
\hline T3/T4 vs. T1/T2 & $2.840(0.89229 .040)$ & 0.770 & - & - \\
\hline \multicolumn{5}{|l|}{ Lymph node metastasis } \\
\hline Positive vs. Negative & $2.344(1.482-3.706)$ & $<0.001$ & $1.355(0.825-2.225)$ & 0.229 \\
\hline \multicolumn{5}{|l|}{ Distant metastasis } \\
\hline Positive vs. Negative & $1.931(0.98023 .805)$ & 0.057 & - & - \\
\hline \multicolumn{5}{|l|}{ CDK10 expression } \\
\hline Negative vs. Positive & $3.188(1.964-5.175)$ & $<0.001$ & $1.604(1.011-2.543)$ & 0.045 \\
\hline
\end{tabular}

HR, hazard ratio; CI, confidence interval.

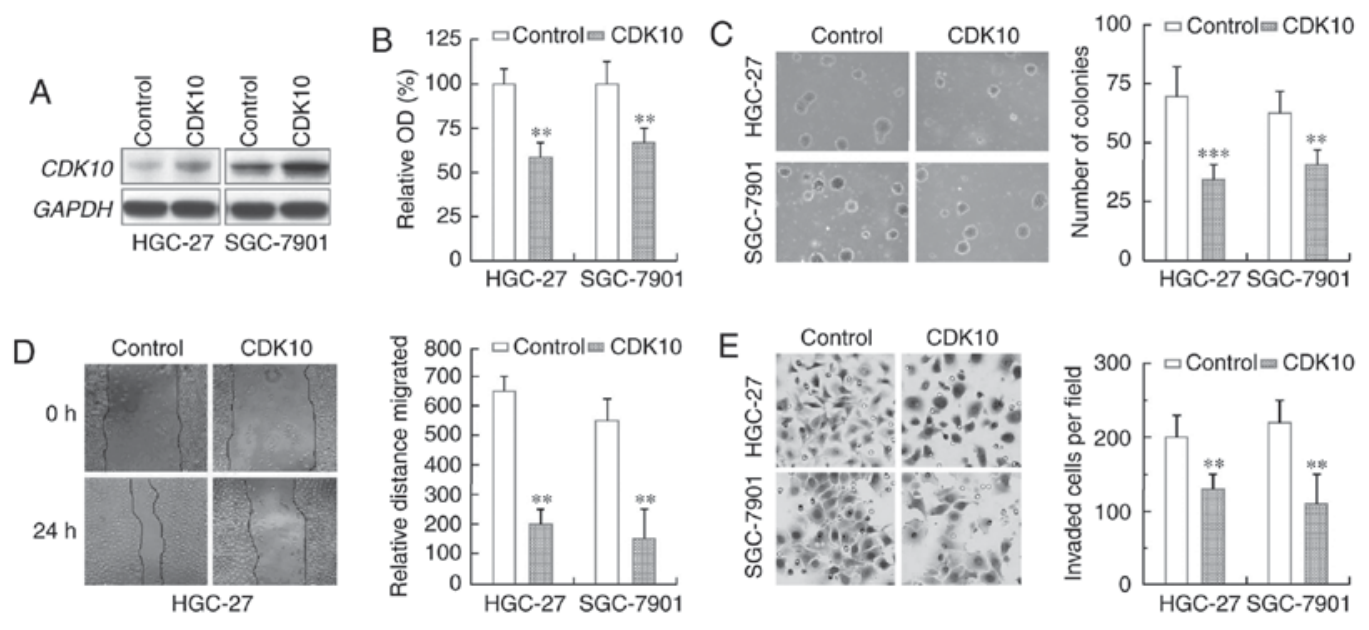

Figure 4. Ectopic CDK10 expression inhibits gastric cancer cell proliferation and migration-invasion. (A) CDK10 Overexpression in HGC-27 and SGC-7901 cells as indicated by immunoblot analysis. CDK10 Overexpression inhibits HGC-27 and SGC-7901 cell proliferation determined using MTT (B) and colony formation (C) assays. (D) CDK10 overexpression inhibits migration of HGC-27 and SGC-7901 cells in a wound healing assay. (E) CDK10 overexpression reduces invasive abilities of HGC-27 and SGC-7901 cells using a Matrigel assay. Each experiment was repeated at least three times. Control, empty vector-transfected control group. ${ }^{* *} \mathrm{P}<0.01,{ }^{* * *} \mathrm{P}<0.001$, compared with the control group. CDK10, cyclin-dependent kinase 10; OD, optical density.

knockdown exhibited a consistent and significant increase in cell proliferation and colony formation, as compared to those cells transfected with non-specific control siRNA (Fig. 5B and C). Further, the inhibitory effect of CDK10 on cell invasion was confirmed using a Matrigel assay. The results showed that HGC-27 and SGC-7901 cells with CDK10 knockdown showed a significant increase in cell invasive capacity compare to their respective control cells (Fig. 5D). Together, these findings 

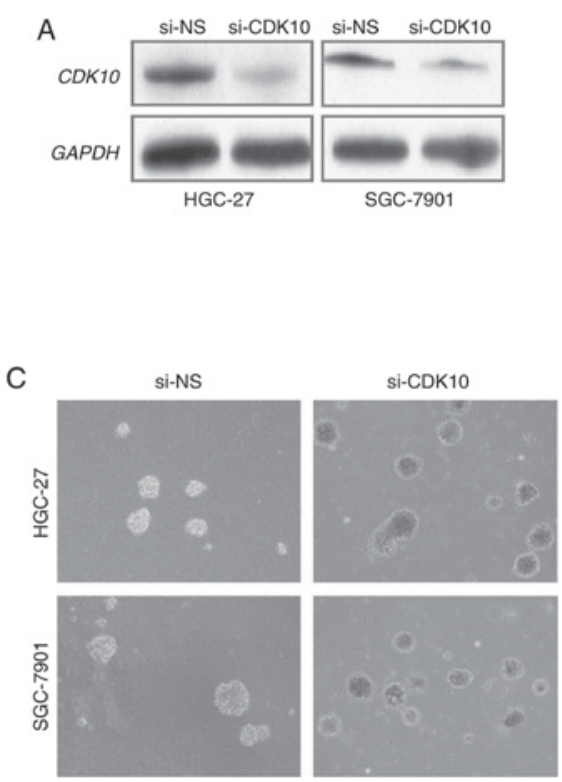

$\mathrm{D}$
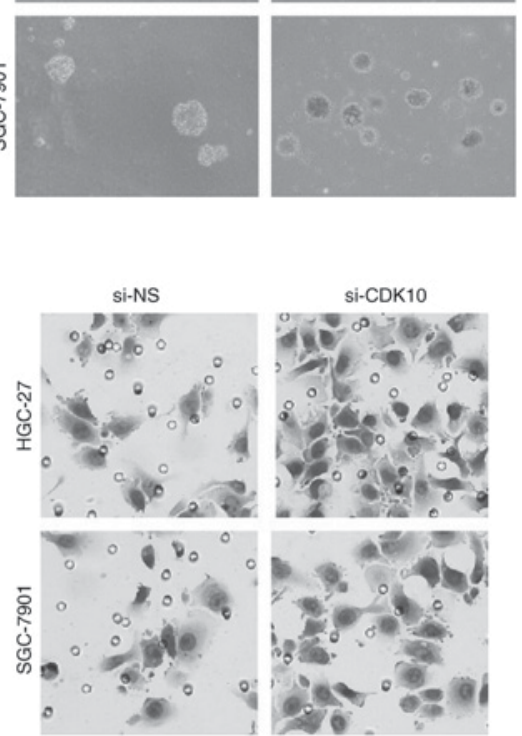
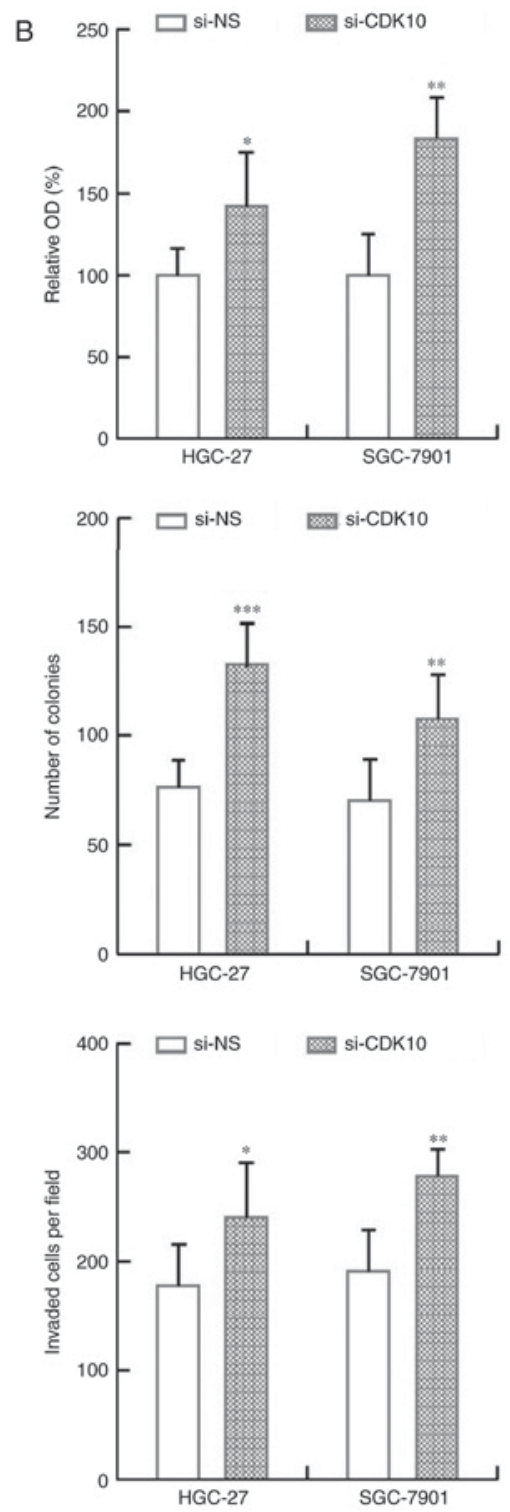

Figure 5. Knockdown of CDK10 promotes gastric cancer cell growth and invasion. (A) Knockdown of CDK10 in HGC-27 and SGC-7901 cells as indicated by immunoblot analysis. Knockdown of CDK10 promotes HGC-27 and SGC-7901 cell proliferation assessed using MTT (B) and a colony formation (C) assays. (D) Knockdown of CDK10 promotes the invasive ability of HGC-27 and SGC-7901 cells using a Matrigel assay. Each experiment was repeated at least three times. si-NS, non-specific control siRNA. ${ }^{*} \mathrm{P}<0.05,{ }^{* *} \mathrm{P}<0.01,{ }^{* * *} \mathrm{P}<0.001$, compared with the si-NS group. CDK10, cyclin-dependent kinase 10 ; OD, optical density.

clearly demonstrate that knockdown of CDK10 promotes the proliferation and the migration-invasion of gastric cancer cells.

\section{Discussion}

We were encouraged to study the CDK10 gene because it is frequently listed in the genes with their expression correlated with cancer patients survival rate $(7,8,12)$. Our present study is to understand the relationship between CDK10 gene expression profiles and cancer outcomes, and discover new biomarkers for diagnosis and prognosis, as well as new therapeutic targets. In our study, we provided the comprehensive analysis of CDK10 in 128 samples from primary gastric cancer patients and investigated the biological function of CDK10 in gastric cancer cells. Our data not only demonstrate that CDK10 expression is down-regulated in gastric cancers and highly associated with patient survival, consistent with our recent study of CDK10 in breast cancer (7), but also show the correlation of CDK10 expression with gastric cancer malignancy further supported by our biological studies. Therefore, our study emphasizes the critical role of CDK10 as a prognostic biomarker for gastric cancer with great therapeutic promise.

Although multiple CDK proteins, particularly those with great therapeutic promise, have been evaluated for prognosis and cancer therapy (19-21), studies on CDK10 have so far only focused on several cancer types. Our current analysis in gastric cancer documents that a connection exists between negative CDK10 expression with severe tumor progression phenotype, including advanced tumor stage, lymph node and distant metastasis, and poor tumor differentiation. These results are similar to the earlier reports on biliary tract cancer and hepatocellular carcinoma, as well as our more recent report in breast cancer $(7,14,15)$. However, in our study we failed to discover that $\mathrm{CDK}$ expression was significantly correlated with tumor 
invasion depth. One of possibilities may be due to the actual composition of samples in which $93.0 \%$ (119/128) were T3/T4 samples, whereas T1/T2 samples were barely $7.0 \%(9 / 128)$. In addition, our data suggests that the expression of CDK10 may serve as an independent prognostic biomarker for the favorable OS, consistent with our report on breast cancer (7). Overall, these observations indicate that CDK10 may be an important factor in regulating gastric cancer development and progression.

CDK10, a member of the Cdc2-related kinase, can identify and activate cyclin M (22). Silencing of CDK10 increases the activation of MAPK pathway drove by ETS2, which confers tamoxifen resistance to breast cancer cells $(12,13)$. To tease out the function of CDK10 in gastric cancer, its expression was restored in gastric cancer cells and its influence on cell growth and migration-invasion were assessed. Over-expression of CDK10 led to a significant decrease in cell growth and migration-invasion activities in vitro; and vice versa, silencing of CDK10 enhanced gastric cancer cell proliferation, migration and invasion. These observations are concordant with the previous reports on other cancer types $(14,15)$, and also support our results in immunohistochemistry analysis in which loss of CDK10 expression was closely correlated with frequent lymph node metastasis and advanced tumor stage. Our data suggest that the CDK10 expression may influence and directly correlate with aggressive behavior of gastric cancer cells.

Although our study has some limitations to generalize the results, to the best of our knowledge, our data provided the first prognostic evidence that CDK10 may function as a tumor suppressor in gastric cancer. In our future study, we will increase the sample number and perform more detailed analyses to validate the clinical value of CDK10 for gastric cancer therapy. Additionally, the precise signaling pathways and molecular mechanism underlying CDK10 involvement in the tumorigenesis and progression of gastric cancer deserves further exploration.

Taken together, our data suggest that silencing of CDK10 is a molecular hallmark of gastric cancer progression. Down-regulation of CDK10 is correlated with aggressive cancer characteristics mainly relevant to metastasis and poor overall survival. We conclude that CDK10 may serve as an independent marker for gastric cancer prognosis that holds great promise to against this malignancy.

\section{Acknowledgements}

This study was supported in part by the Science and Technology Planning Project of Henan Province, China (142102310464) and the Key Research Foundation of Higher Education of Henan Province, China (15B320003). No additional external funding received for this study. The funders had no role in study design, data collection and analysis, decision to publish, or preparation of the manuscript.

\section{References}

1. Crew KD and Neugut AI: Epidemiology of gastric cancer. World J Gastroenterol 12: 354-362, 2006.

2. Jemal A, Siegel R, Ward E, Murray T, Xu J and Thun MJ: Cancer statistics, 2007. CA Cancer J Clin 57: 43-66, 2007.
3. Yoo CH, Noh SH, Shin DW, Choi SH and Min JS: Recurrence following curative resection for gastric carcinoma. Br J Surg 87: 236-242, 2000.

4. Chen CN, Lin JJ, Chen JJ, Lee PH, Yang CY, Kuo ML, Chang KJ and Hsieh FJ: Gene expression profile predicts patient survival of gastric cancer after surgical resection. J Clin Oncol 23: 7286-7295, 2005

5. Jian P, Yanfang T, Zhuan Z, Jian W, Xueming Z and Jian N: MMP28 (epilysin) as a novel promoter of invasion and metastasis in gastric cancer. BMC Cancer 11: 200, 2011.

6. You Y, Yang W, Wang Z, Zhu H, Li H, Lin C and Ran Y: Promoter hypermethylation contributes to the frequent suppression of the CDK10 gene in human nasopharyngeal carcinomas. Cell Oncol (Dordr) 36: 323-331, 2013.

7. You Y, Li H, Qin X, Zhang Y, Song W, Ran Y and Gao F: Decreased CDK10 expression correlates with lymph node metastasis and predicts poor outcome in breast cancer patients-a short report. Cell Oncol (Dordr) 38: 485-491, 2015.

8. Hong CQ, Zhang F, You YJ, Qiu WL, Giuliano AE, Cui XJ, Zhang GJ and Cui YK: Elevated Clorf63 expression is correlated with CDK10 and predicts better outcome for advanced breast cancers: A retrospective study. BMC Cancer 15: 548, 2015.

9. Graña X, Claudio PP, De Luca A, Sang N and Giordano A: PISSLRE, a human novel CDC2-related protein kinase. Oncogene 9: 2097-2103, 1994.

10. Brambilla R and Draetta G: Molecular cloning of PISSLRE, a novel putative member of the cdk family of protein serine/threonine kinases. Oncogene 9: 3037-3041, 1994.

11. Li S, MacLachlan TK, De Luca A, Claudio PP, Condorelli G and Giordano A: The cdc-2-related kinase, PISSLRE, is essential for cell growth and acts in $\mathrm{G} 2$ phase of the cell cycle. Cancer Res 55: 3992-3995, 1995.

12. Iorns E, Turner NC, Elliott R, Syed N, Garrone O, Gasco M, Tutt AN, Crook T, Lord CJ and Ashworth A: Identification of CDK10 as an important determinant of resistance to endocrine therapy for breast cancer. Cancer Cell 13: 91-104, 2008.

13. Khanal P, Yun HJ, Lim SC, Ahn SG, Yoon HE, Kang KW, Hong R and Choi HS: Proyl isomerase Pin1 facilitates ubiquitin-mediated degradation of cyclin-dependent kinase 10 to induce tamoxifen resistance in breast cancer cells. Oncogene 31: 3845-3856, 2012.

14. Yu JH, Zhong XY, Zhang WG, Wang ZD, Dong Q, Tai S, Li H and Cui YF: CDK10 functions as a tumor suppressor gene and regulates survivability of biliary tract cancer cells. Oncol Rep 27: 1266-1276, 2012

15. Zhong XY, Xu XX, Yu JH, Jiang GX, Yu Y, Tai S, Wang ZD and Cui YF: Clinical and biological significance of Cdk10 in hepatocellular carcinoma. Gene 498: 68-74, 2012.

16. You Y, Yang W, Qin X, Wang F, Li H, Lin C, Li W, Gu C, Zhang Y and Ran Y: ECRG4 acts as a tumor suppressor and as a determinant of chemotherapy resistance in human nasopharyngeal carcinoma. Cell Oncol (Dordr) 38: 205-214, 2015.

17. You Y, Li H, Qin X, Ran Y and Wang F: Down-regulated ECRG4 expression in breast cancer and its correlation with tumor progression and poor prognosis-A short Report. Cell Oncol (Dordr) 39: 89-95, 2016.

18. Lin C, Xin S, Qin X, Li H, Lin L and You Y: Zoledronic acid suppresses metastasis of esophageal squamous cell carcinoma cells through upregulating the tight junction protein occludin. Cytotechnology 68: 1233-1241, 2016.

19. Mahale S, Bharate SB, Manda S, Joshi P, Jenkins PR, Vishwakarma RA and Chaudhuri B: Antitumour potential of BPT: A dual inhibitor of cdk4 and tubulin polymerization. Cell Death Dis 6: e1743, 2015.

20. VanArsdale T, Boshoff C, Arndt KT and Abraham RT: Molecular pathways: Targeting the cyclin D-CDK4/6 axis for cancer treatment. Clin Cancer Res 21: 2905-2910, 2015.

21. Feng Z, Xu S, Liu M, Zeng YX and Kang T: Chk1 inhibitor Gö6976 enhances the sensitivity of nasopharyngeal carcinoma cells to radiotherapy and chemotherapy in vitro and in vivo. Cancer Lett 297: 190-197, 2010.

22. Guen VJ, Gamble C, Flajolet M, Unger S, Thollet A, Ferandin Y, Superti-Furga A, Cohen PA, Meijer L and Colas P: CDK10/cyclin $\mathrm{M}$ is a protein kinase that controls ETS2 degradation and is deficient in STAR syndrome. Proc Natl Acad Sci USA 110: 19525-19530, 2013. 